\title{
How palmitoylation affects trafficking and signalling of membrane receptors
}

\author{
Maxime Jansen and Bruno Beaumelle\# \\ Institut de Recherche en Infectiologie de Montpellier (IRIM), UMR9004-Université de \\ Montpellier-CNRS, 1919 route de Mende, 34293 MONTPELLIER, France
}

maxime.jansen@irim.cnrs.fr and bruno.beaumelle@irim.cnrs.fr

\# Corresponding author

\begin{abstract}
S-acylation (or palmitoylation) is a reversible post-translational modification (PTM) that modulates protein activity, signalization and trafficking. Palmitoylation was found to significantly impact the activity of various membrane receptors involved in either pathogen entry, such as CCR5 (for HIV) and anthrax toxin receptors, cell proliferation (epidermal growth factor receptor), cardiac function ( $\beta$-Adrenergic receptor) or synaptic function (AMPA receptor). Palmitoylation of these membrane receptors indeed affects not only their internalization, localization and activation, but also other PTMs such as phosphorylation. In this review we discuss recent results showing how palmitoylation differently affects the biology of these membrane receptors.
\end{abstract}

Running title palmitoylation of membrane receptors

Keywords palmitoylation, traffic, CCR5, Adrenergic receptor, EGFR, anthrax receptor, AMPAR.

Published by Biology of the Cell (Wiley) as https://doi.org/10.1111/boc.202100052 On line 4 Nov 2021 


\section{Abbreviations}

AMPAR,a-amino-3-hydroxy-5-methyl 4-isoxazolepropionic acid-type receptor; ANTXR, anthrax toxin receptor; AR, $\beta$ Adrenergic receptors; APT, acylprotein thioesterase; BACE1, $\beta$-Site APP cleaving enzyme 1; CCR5, C-C chemokine receptor type 5; CME, clathrinmediated endocytosis; CMG2, capillary morphogenesis gene 2; CNS, central nervous system; DHHC, Lys-His-His-Cys; EF, Edema Factor; EGF, epidermal growth factor; EGFR, EGF receptor; ER, endoplasmic reticulum; FASN, fatty acid synthase; GPCR, Gprotein coupled receptor; LF, Lethal Factor; mtEGFR, mitochondrial EGFR; NMDAR, Nmethyl-D-aspartate-type receptor; PA, Protective Antigen; pAKT, Phosphorylated AKT; PAT, protein acyl transferase; PTM, post-translational modification; TEM8, tumor endothelial marker 8; TMD, transmembrane domain; zDHHC, zinc finger DHHC-type.

\section{Introduction}

More than 200 different post-translational modifications (PTM) have been reported to date (Zmuda \& Chamberlain, 2020). There are different types of PTM involving the covalent addition of fatty acids to proteins. They are S-acylation, N-terminal acylation, and O-acylation (Linder and Deschenes, 2007). S-palmitoylation corresponds to the addition of a fatty acid, most often palmitate, on the $\mathrm{SH}$ group of a cysteine. Palmitoylation and $\mathrm{S}$ acylation refer to the same PTM because most protein acyl transferases (PATs) that attach fatty acids to the $\mathrm{SH}$ group of cysteine residues prefer to use palmitoyl-CoA as a substrate, and palmitate is one of the most abundant fatty acid in cells (Lemonidis et al., 2017). Palmitoylation is reversible, with depalmitoylation being performed by acyl-protein thioesterases (APTs) (Linder and Deschenes, 2007; Won et al., 2018). This is in contrast with myristoylation that results in the formation of an essentially irreversible amide link between the carboxyl group of a myristate and the $\mathrm{N}$-terminus of the protein (Aicart-Ramos et al., 2011). This reversibility therefore provides the cell with the possibility to regulate protein function and/or localization via palmitoylation.

In mammals there are 23 different PATs catalyzing S-palmitoylation (Ko \& Dixon, 2018; Zmuda \& Chamberlain, 2020). These enzymes are zinc finger Lys-His-His-Cys (DHHC)-type (zDHHC), also called DHHC or palmitoyl S-acyltransferases. They are polytopic membrane proteins and most of them are located in the ER or the Golgi membrane. Depending on cell types, 2-3 of them only localize to the plasma membrane (Ko \& Dixon, 2018; Chopard et al, 2018). 
Currently, around 4,000 theoretically palmitoylated proteins were reported in human and mouse (Blanc et al., 2019). Among them, the S-acylation of approximatively 350 human proteins was demonstrated (Zmuda \& Chamberlain, 2020). Interestingly, among 299 cancer drivers identified in humans, 79 are palmitoylated (Ko \& Dixon, 2018). Both cytosolic and membrane proteins were found to be palmitoylated (Chamberlain and Shipston, 2015).

Palmitoylation of a membrane protein can affect its localization and trafficking for instance from the Golgi apparatus to the plasma membrane as for c-Met (Coleman et al., 2016), or from the plasma membrane to the nucleus or the mitochondria like for EGFR (Bollu et al., 2014). Palmitoylation can also affect the stability of some membrane proteins. This is the case for some DHHC enzymes (Zmuda \& Chamberlain, 2020).

Most often, because palmitoylation stabilizes membrane interaction, addition of the palmitate group can change the conformation of the cytosolic loop or tail housing the palmitoylated cysteine. For instance, the C-terminal tails of AMPAR or NMDAR (two ionotropic glutamate receptors) are palmitoylated, enabling to attach the receptor tail to the plasma membrane, thereby enabling their interaction with downstream partners that are important for the signalling activity of these receptors (Hayashi, 2021).

In this review we focused on the impact of palmitoylation on signalization and function of different membrane receptors: CCR5, $\beta$-adrenergic receptors (AR), EGFR, anthrax receptors, and AMPAR. These different membrane receptors fulfill different functions, but their activity, trafficking, or maturation were found to be regulated by palmitoylation. We will see how palmitoylation affects the interaction between these receptors and their ligand(s), the maturation and intracellular localization of these receptors, as well as the regulation of other PTMs like phosphorylation, and finally receptor signalling. Interestingly, depending on membrane receptors, palmitoylation can sometime have opposite effects on their activity. 


\section{CCR5}

\section{$\underline{\text { Generalities about CCR5 }}$}

The C-C chemokine receptor type 5 (CCR5) is a G-protein coupled receptor (GPCR) that is considered as a non-essential protein (Jiao et al., 2019), probably because of redundancy in the chemokine network (Bennetts et al., 1997). CCR5 is activated by several ligands such as CCL3 (MIP-1a), CCL3L1, CCL4 (MIP-1b), CCL5 (RANTES), CCL8 (MCP-2), CCL11, CCL13 (MCP-4), or CCL16 (HCC-4). CCR5 interact with four different G proteins: $G_{\alpha i}, G_{\alpha q}, G_{\beta}$ and $G_{\gamma}$. Upon CCR5 activation, these $G$ proteins dissociate from the receptor and activate several signalization pathways that induce a large number of cell responses (Jiao et al., 2019; Wu and Yoder, 2009). Gaq activates the PLC $\beta$ pathway that generates IP3, enabling $\mathrm{Ca}^{2+}$ release from intracellular stores, inducing PYK2, ERK1/2, P38, and the JNK pathway that triggers cell activation and proliferation. PLC $\beta$ also produces DAG, activating PKC and promoting CCR5 phosphorylation. Gai activates the PI$3 K$ / PKB/ Akt pathway inducing protein synthesis and cell proliferation (Hemmings and Restuccia, 2012). $G_{\beta \gamma}$ indirectly activates the RhoA family of small GTPases (Rac/cdc42/RhoA) that remodel the cell cytoskeleton by affecting both actin and tubulin polymerisation. The JAK/STAT pathway can also be activated by MIP-1a and MIP-1b (Jiao et al., 2019; Sanchooli et al., 2014) (Figure 1c).

CCR5 plays a key role in HIV-1 infection. Indeed, individuals who are homozygous for the CCR5 $\triangle 32$ mutation that prevents CCR5 ectopic expression are essentially resistant to HIV infection (Brelot and Chakrabarti, 2018). For HIV-1 entry, the viral glycoproteins gp41 and gp120 sequentially interact with the receptor CD4 and CCR5 that acts as a coreceptor (Kasama et al., 2006) (Figure 1b). CCR5 is also involved in different types of lymphoma and many other cancers such as breast, prostate, colorectal, heart, neck and gastric cancers (Gao and Fish, 2018; Jiao et al., 2019). Moreover, CCR5 plays a role in the regulation of activation and migration of immune cells during viral infection (Sanchooli et al., 2014).

CCR5 is internalized by both clathrin- and caveolae-dependent pathways. Endocytosed CCR5 is targeted to recycling endosomes together with the transferrin receptor and is then recycled (Mueller et al., 2002; Signoret et al., 2000) (Figure 1c). 


\section{Palmitoylation of CCR5}

CCR5 is palmitoylated on three cysteine residues: C321, C323, and C324; these neighbouring palmitoylated sites are in the C-terminal tail of CCR5 (Blanpain et al., 2001; Kraft et al., 2001) (Figure 1a). CCR5 palmitoylation was first found to play a crucial role for the interaction between CCR5 and cholesterol molecules that is required for CCR5 localization into plasma membrane raft domains. This localization is needed for CCR5 to bind MIP-1b and elicit calcium mobilization (Kraft et al., 2001; Nguyen and Taub, 2002). In fact, when CCR5 palmitoylation is inhibited, CCR5 localization into rafts is lost thereby preventing internalization of CCR5 by caveolae-related structures (Blanpain et al., 2001; Kraft et al., 2001; Venkatesan et al., 2003). Upon ligand (RANTES) binding or PMA treatment, CCR5 becomes activated and is phosphorylated on four serine residues: S336, S337, S342, and S349 (Kraft et al., 2001). These phosphorylations are not observed for unpalmitoylated CCR5 (Kraft et al., 2001). Moreover, inhibition of palmitoylation affects CCR5-induced signalization. For instance, while RANTES or MIP-1 $\beta$ rapidly induce a strong rise in intracellular calcium concentration, this increase was significantly delayed in cells expressing unpalmitoylated CCR5 (Blanpain et al., 2001; Kraft et al., 2001).

Several groups showed that inhibition of CCR5 palmitoylation using point mutations or chemicals prevents its transport to the plasma membrane (Blanpain et al., 2001; Boncompain et al., 2019; Percherancier et al., 2001). Moreover, palmitoylation-deficient CCR5 mutants showed a 3-fold reduced half-life compared with WT. The degradation of unpalmitoylated CCR5 was inhibited by bafilomycin A1 (an inhibitor of vacuolar-type $\mathrm{H}^{+}$ATPase), indicating that it takes place in lysosomes (Percherancier et al., 2001). Although drugs (cadmium chloride and zinc pyrithione) that inhibit CCR5 palmitoylation decreased by $\sim 20 \%$ CCR5 cell surface expression, they caused a stronger reduction (by 30 to $80 \%$ ) in HIV entry and multiplication. These results indicate the importance of CCR5 ectopic expression and palmitoylation for HIV-1 entry (Boncompain et al., 2019).

Both DHHC3 and DHHC7, that localize to the Golgi apparatus (Chopard et al., 2018; Ohno et al., 2006) were found to palmitoylate CCR5. In fact the autopalmitoylation of these PATs was inhibited by $\mathrm{CdCl}$ and $\mathrm{Zn}$ pyrithione, indicating that these drugs might affect CCR5 palmitoylation by inhibiting the DHHCs responsible for this PTM (Boncompain et al., 2019). 


\section{$\beta$-Adrenergic receptors}

\section{$\underline{\text { Generalities about } \beta \text {-Adrenergic receptors }}$}

The $\beta$-Adrenergic receptors ( $\beta$-AR), are one of two subfamilies of $A R$, together with $\alpha-A R$. The $\beta-A R$ subfamily is composed of three subtypes: $\beta 1-A R, \beta 2-A R$, and $\beta 3-A R$. These three receptor subtypes are GPCR and interact with different subunits of $G \alpha$ via the third inner loop of every $\beta$-AR (Baker, 2014). The three $\beta$-AR are expressed in the heart (Imbrogno et al., 2015), and in the lung (Nilsson et al., 2020), but the $\beta 1$ subtype is the prominent one (Lohse et al., 2003).

In the heart, ligands (adrenalin or noradrenalin) induce the interaction of $\beta-A R$ with $G$ proteins, triggering the production of CAMP that results in the activation of ERK1/2 and PKA by phosphorylation. Phosphorylated PKA triggers $\mathrm{Ca} 2+$ liberation, increasing contraction rate. Hence, chronic hyperactivation of $\beta-A R$ can induce heart failure (Baker, 2014) and adrenergic antagonists are widely used in heart failure therapy (Lohse et al., 2003). Moreover, $\beta_{2}$-AR signalling is involved in the development of lung cancer (Nilsson et al., 2020).

\section{Palmitoylation of $\beta_{1}-A R$}

For $\beta_{1}-\mathrm{AR}$, palmitoylation sites are a subject of controversy. It is indeed not clear whether $\beta_{1}$-AR is palmitoylated on C358 only (Warne et al., 2009), or also on C392, C393, and C414 (Zuckerman et al., 2011) (Figure 2a). Two groups observed that DHHC21 is responsible for $\beta_{1}$-AR palmitoylation (Marin et al., 2016; Yang et al., 2021). DHHC21 essentially localizes to the Golgi apparatus (Chopard et al., 2018; Fernández-Hernando et al., 2006; Tonn Eisinger et al., 2018).

Although palmitoylation does not affect the half-life or steady-state levels of $\beta_{1}-A R$, palmitoylation on $\mathrm{C} 414$ is required for efficient receptor endocytosis following agonist stimulation. In fact, palmitoylation on $\mathrm{C} 414$ only was found to affect $\beta_{1}$-AR receptor activities. Moreover, this $\mathrm{C} 414$ palmitoylation site showed a turnover 2.5 -fold faster than other palmitoylation sites (Zuckerman et al., 2011). Regarding the mechanistic, it was suggested that $\mathrm{C} 414$ palmitoylation affects the conformation of the $\mathrm{C}$-terminal domain of $\beta_{1}$-AR, impeding the interaction between $\beta_{1}$-AR and the $G$ protein (Zuckerman et al., 2011). 


\section{Palmitoylation of $\beta_{2}-A R$}

For $\beta_{2}-A R$, two palmitoylation sites on C265 and C341 were identified (Adachi et al., 2016) (Figure 2a). Three different PATs, DHHC9, DHHC14, and DHHC18 are able to palmitoylate $\beta_{2}-A R$ in the Golgi apparatus, while APT-1 promotes $\beta_{2}-A R$ depalmitoylation (Adachi et al., 2016). Absence of palmitoylation reduced agonist-induced cAMP production (Liu et al., 2012), but, surprisingly, $\beta_{2}$-AR palmitoylation inhibited its PKA-dependent phosphorylation on serines S261 and S262 (Liu et al., 2012; Loisel et al., 1996; Moffett et al., 2001). In fact, both $\beta_{2}-A R$ phosphorylation and palmitoylation are important to activate the GPCR pathway following agonist stimulation (Adachi et al., 2016; Liu et al., 2012;

Moffett et al., 2001). This activation induces the phosphorylation of ERK1/2 (pERK1/2) and PKA. Inhibition of $\beta_{2} A R$ palmitoylation leads to higher PKA activity and increased myocyte contraction rates that can cause heart failure (Adachi et al., 2016; Liu et al., 2012; Loisel et al., 1996). Palmitoylation is thus required for the correct and regulated activity of $\beta_{2} A R$.

\section{Palmitoylation of $\beta_{3}-A R$}

Mouse $\beta_{3}-A R$ is palmitoylated on $\mathrm{C} 358$ at a canonical site within the $C$-terminal tail of the receptor, while human $\beta_{3}-A R$ is not only palmitoylated on this site (C361 and C363), but also on $\mathrm{C} 153$ and C292 that are within other intracellular loops (Adachi et al., 2019) (Figure 2a). Palmitoylation of human but not mouse $\beta_{2}-A R$ is important for CAMP production. Along the same lines, palmitoylation of human but not mouse $\beta_{3}-A R$ was found to be critical for its ectopic expression and stability (Adachi et al., 2019).

\section{EGFR}

\section{$\underline{\text { Generalities about EGFR }}$}

The epidermal growth factor (EGF) receptor (EGFR) is a member of the ErbB receptor family (Caldieri et al., 2018). EGFR is a key receptor that regulates numerous processes such as cell proliferation, migration, survival, development, differentiation and apoptosis (Caldieri et al., 2018; Romano and Bucci, 2020). Activation of EGFR by EGF promotes the phosphorylation of AKT. pAKT inhibits autophagy and reduces GSK3 activity, enhancing $\beta$-catenin signalling (Caldieri et al., 2018; Termini and Gillette, 2017) (Figure 3b). The intracellular pathway of activated EGFR shows some unusual specificities. First, depending on the EGF dose, EGFR is internalized using clathrin- 
dependent or -independent pathways. Activated EGFR can then translocate to different intracellular compartments such as the nucleus or, more surprisingly, the mitochondria (Wang and Hung, 2012). EGFR is well known to be a driver of tumorigenesis. Both EGFR activation and amplification are observed in many cancers (Ko and Dixon, 2018). EGFR is also involved in the development of neurologic diseases. For instance, a reduction in EGFR signalization in the nervous system is thought to be involved in Parkinson disease, while inhibitors of EGFR activation were found beneficial to prevent neuroinflammation during Alzheimer disease (Romano and Bucci, 2020).

\section{EGFR Palmitoylation}

Two palmitoylation sites at $\mathrm{C} 781$ and $\mathrm{C} 797$ were found in the mitochondrial EGFR (mtEGFR) (Bollu et al., 2014), while 3 different palmitoylation sites at C1025, C1034, and C1122 were identified for plasma membrane EGFR (pmEGFR) (Runkle et al., 2016) (Figure 3a). It is not clear why palmitoylation sites differ depending on the subcellular localization of the receptor, since mitochondria do not seem to express any DHHC (Ko and Dixon, 2018).

After activation of pmEGFR by EGF, fatty acid synthase (FASN) is activated, producing palmitate that can activate mtEGFR. Palmitoylation at $\mathrm{C797}$ is required for mtEGFR activation that promotes mitochondria fusion by increasing the levels of the fusion proteins PHB2 and OPA1 (Figure 3b) (Bollu et al., 2014). Mitochondrial fusion is thought to favor mitochondrial function by allowing the spreading of enzymes and metabolites (Westermann, 2012).

Palmitoylation at $\mathrm{C} 1025$ and $\mathrm{C} 1122$ were found to differentially regulate pmEGFR activity. Palmitoylation at $\mathrm{C} 1122$ increases upon pmEGFR stimulation and palmitoylation at this site promotes the turnover of pmEGFR, i.e. its degradation. Palmitoylation of C1025 increases upon receptor activation by EGF, inhibiting receptor binding to the MAPKadaptor protein Grb2 that plays a key role in signal transduction and cell communication. In fact, EGFR palmitoylation reversibly attachs the C-terminal tail of the receptor to the plasma membrane to favor receptor inactivation by endocytosis and downregulation (Runkle et al., 2016). pmEGFR palmitoylation on C1025, C1034, and C1122 is performed by DHHC20 (Kharbanda et al., 2020; Runkle et al., 2016), one of the few DHHCs that localize to the plasma membrane (Chopard et al., 2018; Ko and Dixon, 2018; Ohno et al., 
2006). Interestingly, DHHC20 expression was found to induce cell transformation and, accordingly, it is overexpressed in several human tumors (Draper and Smith, 2010).

pmEGFR palmitoylation has been found to play an important role in cell transformation. This is especially the case for the non-small cell lung cancers which are often characterized by mutations on both EGFR and KRAS. Inhibition of EGFR palmitoylation impairs the recruitment of p85 (the regulatory subunit of PI3K) to the plasma membrane, thereby reducing the activity of the PI3K-Akt pathway, signalling and cell proliferation. Conversely, absence of EGFR palmitoylation increases EGFR binding to Grb2, EGFR basal autophosphorylation, AKT and ERK basal phosphorylation (Runkle et al., 2016). EGFR palmitoylation thus regulates the balance between PI3K and MAPK signalling (Kharbanda et al., 2020). Unpalmitoylated EGFR triggers stronger signalling, promoting cell migration and transformation, but also sensitizes cells to EGFR inhibitors such as Gefitinib (Runkle et al., 2016). Preventing EGFR palmitoylation via knocking down DHHC20, expressing palmitoylation deficient EGFR or pharmacological inhibition of FASN reduced cell proliferation in culture and the growth of tumor cells in mice (Ali et al., 2018; Kharbanda et al., 2020). The inhibition of EGFR palmitoylation thus offers new strategies for cancer treatment (Ko \& Dixon, 2018).

\section{Anthrax receptors}

\section{Properties of Anthrax receptors}

Anthrax is a very serious disease that can be life-threatening if not treated. There are different ways of being infected by the causative agent, i.e. the Gram-positive bacterium, Bacillus anthracis. Indeed, anthrax disease can be cutaneous, gastrointestinal, inhalational, or intravenous. The incubation period can be from less than $24 \mathrm{~h}$ to more than 40 days, depending of the type of infection (Sweeney et al., 2011).

The anthrax toxin is produced by B.anthracis. This toxin is composed of three subunits: the Protective Antigen (PA), the Lethal Factor (LF), and the Edema Factor (EF). PA binds to two main receptors: the tumor endothelial marker 8 (TEM8), also known as ANTXR1, and capillary morphogenesis gene 2 (CMG2), also known as ANTXR2 (Friebe et al., 2016; Savransky et al., 2020). PA is a protein of $83 \mathrm{kDa}$ (PA $\left.{ }^{83}\right)$, but following PA binding to these receptors a furin-like protease cleaves $\mathrm{PA}$ in two fragments, a C-terminal part of $63 \mathrm{kDa}\left(\mathrm{PA}^{63}\right)$ that remains on the receptor, and a $\mathrm{N}$-terminal part of $20 \mathrm{kDa}$ that is released. After PA cleavage, the couple $\mathrm{PA}^{63}$-receptor interacts with 6 other couples $\mathrm{PA}^{63}$. 
receptor, the EF, and the LF. This large complex is recruited to lipid raft domains and receptor ubiquitination then triggers endocytosis by the clathrin pathway (Abrami et al., 2006). Upon exposure to endosomal low $\mathrm{pH}$, the PA-complex forms a pore within the endosome membrane that allows EF and LF to translocate either directly in the cytosol or into vacuoles that can later fuse with the endosome membrane (Friebe et al., 2016). The cytosolic delivery of EF and LF contributes to the disease with the adenylate cyclase EF that increases intracellular cAMP leading to edema, while the endoprotease LF cleaves MEKs to induce necrosis and hypoxia, ultimately affecting cell proliferation, and triggering apoptosis (Friebe et al., 2016; Savransky et al., 2020) (Figure 4b).

\section{Palmitoylation of Anthrax receptors}

The two receptors are palmitoylated at different sites. TEM8 can be palmitoylated at C346, C347, C481, and possibly C521; the palmitoylation sites of CMG2 remain to be identified (Abrami et al., 2006). However, since CMG2 shows a strong homology with TEM8, it seems that that CMG2 can be palmitoylated at cysteine 346, 347, 481, and 485 (Deuquet et al., 2012) (Figure 4a). Contrarily to CCR5 (Venkatesan et al, 2003), TEM8 palmitoylation prevents its localization into raft-like domains of the plasma membrane, and therefore its premature endocytosis and targeting to lysosomes. Accordingly, the lack of palmitoylation reduces the half-life of TEM8 three times and cells expressing palmitoylation-deficient TEM8 are less sensitive to anthrax toxin (Abrami et al., 2006).

Anthrax receptor palmitoylation does not only modulate cell intoxication by the anthrax toxin. Antrx1 et Antxr2 were found to interact with RhoA-GTP to insure the correct orientation of the mitotic spindle during cell-oriented division in the zebrafish. Anthrax receptor palmitoylation is required to allow spindle rotation and oriented mitosis (Castanon et al., 2020).

\section{AMPAR}

\section{Generalities about AMPAR}

There are two main types of glutamate receptors, metabotropic and ionotropic. Ionotropic receptors are ligand-gated ion channels that can be classified in three subtypes, based on their affinity for selective structural analogues of glutamate (Jiang et al., 2006). The $\alpha-$ amino-3-hydroxy-5-methyl-4-isoxazolepropionic acid-type receptor (AMPAR) is the 
principal glutamate-gated ion channel present in the mammalian central nervous system (CNS) (Sohn and Park, 2019). AMPAR is made of four different subunits: GluA1 (or GluR1), GluA2 (or GluR2), GluA3 (or GluR3), and GluA4 (or GluR4) that share 70\% identity. Depending for instance on the CNS region, they assemble to form tetrameric cation channels in different combinations, essentially GluA1/GluA2 or GluA2/GluA3 heterotetramers that are composed of two of each subunits (Figure $5 b$ ). In fact, GluA4 expression is restricted to early postnatal development (Jiang et al., 2006).

AMPAR plays an important role in synaptic transmission, synaptic plasticity, as well as for learning and memory cognitive functions in the brain. Defective AMPAR trafficking has been observed in neurodegenerative diseases such as Alzheimer, schizophrenia, intellectual disability and neuronal ceroid lipofuscinosis (Itoh et al., 2018; Sohn and Park, 2019). For instance, in Alzheimer's disease, AMPAR seems to be more efficiently internalized, causing loss of dendritic spines and inhibition of hippocampal long-term potentiation (Jiang et al., 2006).

\section{Palmitoylation of AMPAR}

Each subunit of AMPAR is palmitoylated at two different sites, after the second and the fourth transmembrane domain (TMD). GluA1 is palmitoylated at C585 and C811, GluA2 at C610 and C836, GluA3 at C615 and C841, and GluA4 at C611 and C817 (Hayashi et al., 2005) (Figure 5a). DHHC3 (also called GODZ) localizes to the Golgi apparatus (Chopard et al., 2018; Fukata and Fukata, 2010; Ohno et al., 2006) and enables palmitoylation of GluA1 and GluA2 after TMD2 (Gratacòs-Batlle et al., 2018; Hayashi et al., 2005; Spinelli et al., 2017; Yang et al., 2009). GODZ-mediated palmitoylation of GluA2 promotes its accumulation at the Golgi, suggesting that depalmitoylation triggers receptor trafficking to the cell surface, although the steady state surface level of the AMPAR is not affected by palmitoylation (Hayashi et al., 2005). AMPAR intracellular accumulation is observed under high fat diet conditions that induce FoxO3a-mediated overexpression of DHHC3, promote AMPAR palmitoylation thereby inhibiting its transport to the plasma membrane and affecting AMPAR current amplitudes (Spinelli et al., 2017). Conversely, lack of GluA1 palmitoylation can lead to hyperexcitability in the cerebrum, perturbating network stability and resulting in epileptic seizures (Itoh et al., 2018). 
Palmitoylated AMPAR is susceptible to AMPA and NMDA-induced internalization, while depalmitoylation increases AMPAR affinity for protein $4.1 \mathrm{~N}$, spectrin and actin, favoring AMPAR residence at the plasma membrane (Hayashi et al., 2005). Hence palmitoylation regulates not only receptor exit from the Golgi but also receptor half-life at the cell surface. Moreover, when AMPAR is palmitoylated, its phosphorylation level is low and vice-versa, indicating that AMPAR palmitoylation might inhibit proximal phosphorylation (Itoh et al., 2018; Lin et al., 2009; Spinelli et al., 2017). For instance, depalmitoylation of Cys811 (GluA1) favors PKC-mediated phosphorylation of nearby Ser816 (Lin et al., 2009).

\section{Conclusion}

Protein palmitoylation strongly affects the localization and therefore the biological activity of cytosolic proteins, stabilizing their interaction with membranes (Chopard et al., 2018; Salaun et al., 2010). Regarding membrane proteins that are efficiently anchored through one or several membrane spanning domains, the effects of palmitoylation on their localization or activity is often more subtle. In fact, palmitoylation of membrane receptors does not always affect their signalization, trafficking or interaction with cognate ligands. Indeed, for some membrane receptors that are known to be palmitoylated, no evidence was reported until now for a role of palmitoylation on receptor signalization or trafficking. This is the case for instance of $\alpha$-AR (Kennedy and Limbird, 1993) and NMDAR (Xia et al., 2019).

Nevertheless, palmitoylation of several membrane receptors can affect their phosphorylation level. It can either promote it, like for CCR5 (Kraft et al., 2001), or inhibit it, like for $\beta 2-A R$ (Liu et al., 2012), EGFR (Bollu et al., 2015), and AMPAR (Itoh et al., 2018; Lin et al., 2009; Spinelli et al., 2017). Palmitoylation can regulate receptor signalling at different stages, at the receptor level as reviewed here but also at the level of proteins involved in the signalling cascade downstream of the receptor. For instance, $G$ proteins that transmit GPCR signals can themselves be palmitoylated (Marin et al., 2016; Tsutsumi

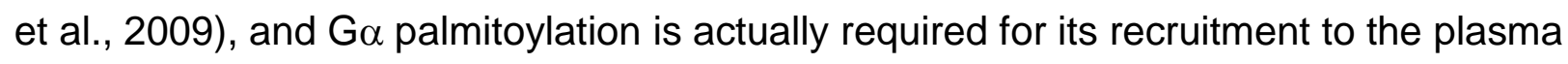
membrane. Moreover, $G$ protein palmitoylation can itself be under the receptor control. Indeed, agonist (isoproterenol) stimulation increases palmitoylation of both $\beta_{2}$-AR (Mouillac et al., 1992) and its associated Go proteins (Mumby et al., 1994). Another example is Akt which acts downstream of EGFR (Runkle et al., 2016) and many other signalling receptors, and that was recently found to be palmitoylated (Blaustein et al., 2021). 
How can receptor palmitoylation regulates the function of these membrane proteins? Several studies on different receptors such as GPCRs (Chini and Parenti, 2009), AMPAR (Hayashi, 2021) and EGFR (Runkle et al., 2016) suggest that palmitoylation likely attach the cytosolic tail or cytosolic loop(s) of the receptor to the membrane so that interaction with the signalling and/or endocytosis machineries is optimal. Nevertheless, for some receptors, palmitoylation prevents the interaction of the receptor cytosolic domain with partners such as Grb2 for EGFR (Runkle et al., 2016) and protein 4.1 for AMPAR (Hayashi et al., 2005). In the case of GPCRs, crystallographic data indicated that palmitoylation additionally enables receptor dimerization (Cherezov et al., 2007).

For the numerous membrane receptors whose PAT was not observed at the plasma membrane, their palmitoylation likely takes place during their transport through the exocytic pathway (ER/Golgi apparatus) where most DHHCs localize to. This specific DHHC localization suggests that receptors such as CCR5, AR or AMPAR are unlikely to be repalmitoylated if their palmitate is removed by an APT at the plasma membrane or during their journey through the endocytic pathway. Conversely, receptors whose DHHC localizes to the plasma membrane can be repalmitoylated following depalmitoylation. For instance DHHC5, a PAT present at the plasma membrane level (Chopard et al., 2018; Ohno et al., 2006) was found to control, together with APT1, the palmitoylation cycle of the transmembrane sodium-calcium exchanger 1, (Gök et al., 2020). Nevertheless, even if their DHHC was not observed at the plasma membrane, some receptors such as $\beta 2-A R$ can undergo agonist-induced palmitoylation (Adachi et al., 2016; Mouillac et al., 1992). Although $\mathrm{DHHC9}, \mathrm{DHHC} 14$, or $\mathrm{DHHC} 18$ that enable $\beta 2-\mathrm{AR}$ palmitoylation are concentrated in the Golgi (Chopard et al., 2018; Ohno et al., 2006), a low level of these enzymes barely detectable by microscopy techniques could be present at the plasma membrane, enabling agonist-induced palmitoylation at this level.

Receptor palmitoylation can affect a large number of diseases. Regarding infectious diseases, receptor palmitoylation can impact pathogen entry and fate. For instance, HIV cannot invade cells that have an unpalmitoylated CCR5 because the latter is retained in the secretory pathway and therefore cannot play its key role in viral entry at the plasma membrane level (Boncompain et al., 2019). In the case of anthrax, palmitoylation of anthrax toxin receptors is required for their endocytosis which is the first step of the cell intoxication (Abrami et al., 2006).

Palmitoylation of membrane receptors also affects numerous non-infectious diseases. Regarding cardiovascular diseases, $\beta_{2}$-AR depalmitoylation increases the heart 
contraction rates and can cause heart failure (Adachi et al., 2016; Liu et al., 2012; Loisel et al., 1996). The relationship between cancer and receptor palmitoylation is well established and was reviewed recently (Ko \& Dixon, 2018). For instance, EGFR depalmitoylation was found to reduce the growth of tumors in mice (Ali et al., 2018; Kharbanda et al., 2020). Regarding CNS diseases, $\beta$-Site APP cleaving enzyme 1 (BACE1), the transmembrane aspartyl protease that initiates $A \beta$ production in Alzheimer disease is palmitoylated (Vetrivel et al., 2009). Unpalmitoylated BACE1 is not targeted to dendritic spines and axons, causing a significant reduction of cerebral amyloid burden in transgenic mice (Andrew et al., 2017). Another example is the AMPAR, since defect in GluA1 palmitoylation is associated with epileptic seizures (Itoh et al., 2018). The role of protein palmitoylation in CNS diseases is discussed in detail elsewhere (Cho and Park, 2016).

Palmitoylation can thus strongly affect the activity of several membrane receptors. More globally the large number of PAT, with $23 \mathrm{zDHHCs}$ identified in the human genome, and the fact that these enzymes are regulated by several PTMs indicate that palmitoylation is an important regulator for the several thousands of human proteins that are thought to be palmitoylated (Blanc et al., 2019; Zmuda and Chamberlain, 2020).

\section{Funding}

This work was funded by the CNRS and the University of Montpellier.

\section{Conflict of interest statement}

The authors declare that they have no conflict of interest. 


\section{Bibliography}

Abrami, L., Leppla, S.H., van der Goot, F.G., 2006. Receptor palmitoylation and ubiquitination regulate anthrax toxin endocytosis. J. Cell Biol. 172, 309-320.

Adachi, N., Hess, D.T., Kaku, M., Ueda, C., Numa, C., Saito, N., 2019. Differential Spalmitoylation of the human and rodent $\beta 3$ adrenergic receptors. J. Biol. Chem. 294, 2569-2578.

Adachi, N., Hess, D.T., McLaughlin, P., Stamler, J.S., 2016. S-Palmitoylation of a Novel Site in the $\beta 2$-Adrenergic Receptor Associated with a Novel Intracellular Itinerary. J. Biol. Chem. 291, 20232-20246.

Aicart-Ramos, C., Valero, R.A., RodriguezCrespo, I., 2011. Protein palmitoylation and subcellular trafficking. Biochim Biophys Acta 1808, 2981-2994.

Ali, A., Levantini, E., Teo, J.T., Goggi, J., Clohessy, J.G., Wu, C.S., Chen, L., Yang, H., Krishnan, I., Kocher, O., Zhang, J., Soo, R.A., Bhakoo, K., Chin, T.M., Tenen, D.G., 2018. Fatty acid synthase mediates EGFR palmitoylation in EGFR mutated non-small cell lung cancer. EMBO molecular medicine 10. e8313.

Andrew, R.J., Fernandez, C.G., Stanley, M., Jiang, H., Nguyen, P., Rice, R.C., BuggiaPrévot, V., De Rossi, P., Vetrivel, K.S., Lamb, R., Argemi, A., Allaert, E.S., Rathbun, E.M., Krause, S.V., Wagner, S.L., Parent, A.T., Holtzman, D.M., Thinakaran, G., 2017. Lack of BACE1 S-palmitoylation reduces amyloid burden and mitigates memory deficits in transgenic mouse models of Alzheimer's disease. Proc. Natl. Acad. Sci. U.S.A. 114, E9665-E9674.

Baker, A.J., 2014. Adrenergic signaling in heart failure: a balance of toxic and protective effects. Pflugers Arch. 466, 1139-1150.

Bennetts, B.H., Teutsch, S.M., Buhler, M.M., Heard, R.N., Stewart, G.J., 1997. The CCR5 deletion mutation fails to protect against multiple sclerosis. Hum. Immunol. 58, 52-59.

Blanc, M., David, F.P.A., van der Goot, F.G., 2019. SwissPalm 2: Protein S-Palmitoylation Database. Methods Mol. Biol. 2009, 203214.
Blanpain, C., Wittamer, V., Vanderwinden, J.M., Boom, A., Renneboog, B., Lee, B., Le Poul, E., El Asmar, L., Govaerts, C., Vassart, G., Doms, R.W., Parmentier, M., 2001. Palmitoylation of CCR5 is critical for receptor trafficking and efficient activation of intracellular signaling pathways. J. Biol. Chem. 276, 23795-23804.

Blaustein, M., Piegari, E., Martínez Calejman, C., Vila, A., Amante, A., Manese, M.V., Zeida, A., Abrami, L., Veggetti, M., Guertin, D.A., van der Goot, F.G., Corvi, M.M., Colman-Lerner, A., 2021. Akt Is S-

Palmitoylated: A New Layer of Regulation for Akt. Front. Cell Dev. Biol. 9, 626404.

Bollu, L.R., Katreddy, R.R., Blessing, A.M., Pham, N., Zheng, B., Wu, X., Weihua, Z., 2015. Intracellular activation of EGFR by fatty acid synthase dependent palmitoylation. Oncotarget 6, 34992-35003.

Bollu, L.R., Ren, J., Blessing, A.M., Katreddy, R.R., Gao, G., Xu, L., Wang, J., Su, F., Weihua, Z., 2014. Involvement of de novo synthesized palmitate and mitochondrial EGFR in EGF induced mitochondrial fusion of cancer cells. Cell Cycle (Georgetown, Tex.) 13, 2415-2430.

Boncompain, G., Herit, F., Tessier, S., Lescure, A., Del Nery, E., Gestraud, P., Staropoli, I., Fukata, Y., Fukata, M., Brelot, A., Niedergang, F., Perez, F., 2019. Targeting CCR5 trafficking to inhibit HIV-1 infection. Sci Adv 5, eaax0821.

Brelot, A., Chakrabarti, L.A., 2018. CCR5 Revisited: How Mechanisms of HIV Entry Govern AIDS Pathogenesis. J. Mol. Biol. 430, 2557-2589.

Caldieri, G., Malabarba, M.G., Di Fiore, P.P., Sigismund, S., 2018. EGFR Trafficking in Physiology and Cancer. Progress in Molecular and Subcellular Biology 57, 235272.

Castanon, I., Hannich, J.T., Abrami, L., Huber, F., Dubois, M., Müller, M., van der Goot, F.G., Gonzalez-Gaitan, M., 2020. Wntcontrolled sphingolipids modulate Anthrax Toxin Receptor palmitoylation to regulate oriented mitosis in zebrafish. Nat. Commun. $11,3317$. 
Chamberlain, L.H., Shipston, M.J., 2015. The physiology of protein S-acylation. Physiol Rev 95, 341-376.

Cherezov, V., Rosenbaum, D.M., Hanson, M.A., Rasmussen, S.G.F., Thian, F.S., Kobilka, T.S., Choi, H.-J., Kuhn, P., Weis, W.I., Kobilka, B.K., Stevens, R.C., 2007. High-resolution crystal structure of an engineered human beta2-adrenergic $\mathrm{G}$ protein-coupled receptor. Science 318, 1258-1265.

Chini, B., Parenti, M., 2009. G-protein-coupled receptors, cholesterol and palmitoylation: facts about fats. J. Mol. Endocrinol. 42, 371379.

Cho, E., Park, M., 2016. Palmitoylation in

Alzheimer's disease and other neurodegenerative diseases. Pharmacological Research 111, 133-151.

Chopard, C., Tong, P.B.V., Tóth, P., Schatz, M., Yezid, H., Debaisieux, S., Mettling, C., Gross, A., Pugnière, M., Tu, A., Strub, J.-M., Mesnard, J.-M., Vitale, N., Beaumelle, B., 2018. Cyclophilin A enables specific HIV-1 Tat palmitoylation and accumulation in uninfected cells. Nat. Commun. 9, 2251.

Coleman, D.T., Gray, A.L., Kridel, S.J., Cardelli, J.A., 2016. Palmitoylation regulates the intracellular trafficking and stability of c-Met. Oncotarget 7, 32664-32677.

Deuquet, J., Lausch, E., Superti-Furga, A., van der Goot, F.G., 2012. The dark sides of capillary morphogenesis gene 2 . EMBO J 31, 3-13.

Draper, J.M., Smith, C.D., 2010. DHHC20: a human palmitoyl acyltransferase that causes cellular transformation. Mol. Membr. Biol. 27, 123-136.

Fernández-Hernando, C., Fukata, M., Bernatchez, P.N., Fukata, Y., Lin, M.I., Bredt, D.S., Sessa, W.C., 2006. Identification of Golgi-localized acyl transferases that palmitoylate and regulate endothelial nitric oxide synthase. J. Cell Biol. 174, 369-377.

Friebe, S., van der Goot, F.G., Bürgi, J., 2016. The Ins and Outs of Anthrax Toxin. Toxins (Basel) 8. 69.

Fukata, Y., Fukata, M., 2010. Protein palmitoylation in neuronal development and synaptic plasticity. Nat. Rev. Neurosci. 11, 161-175.
Gao, D., Fish, E.N., 2018. Chemokines in breast cancer: Regulating metabolism. Cytokine 109, 57-64.

Gök, C., Plain, F., Robertson, A.D., Howie, J., Baillie, G.S., Fraser, N.J., Fuller, W., 2020. Dynamic Palmitoylation of the SodiumCalcium Exchanger Modulates Its Structure, Affinity for Lipid-Ordered Domains, and Inhibition by XIP. Cell Rep. 31, 107697.

Gratacòs-Batlle, E., Olivella, M., SánchezFernández, N., Yefimenko, N., MiguezCabello, F., Fadó, R., Casals, N., Gasull, X., Ambrosio, S., Soto, D., 2018. Mechanisms of CPT1C-Dependent AMPAR Trafficking Enhancement. Front. Mol. Neurosci. 11, 275.

Hayashi, T., 2021. Post-translational palmitoylation of ionotropic glutamate receptors in excitatory synaptic functions. $\mathrm{Br}$. J. Pharmacol. 178, 784-797.

Hayashi, T., Rumbaugh, G., Huganir, R.L., 2005. Differential regulation of AMPA receptor subunit trafficking by palmitoylation of two distinct sites. Neuron 47, 709-723.

Hemmings, B.A., Restuccia, D.F., 2012. PI3KPKB/Akt Pathway. Cold Spring Harb. Perspect. Biol. 4. a011189

Imbrogno, S., Gattuso, A., Mazza, R., Angelone, T., Cerra, M.C., 2015. $\beta 3$-AR and the vertebrate heart: a comparative view. Acta Physiol. 214, 158-175.

Itoh, M., Yamashita, M., Kaneko, M., Okuno, H., Abe, M., Yamazaki, M., Natsume, R., Yamada, D., Kaizuka, T., Suwa, R., Sakimura, K., Sekiguchi, M., Wada, K., Hoshino, M., Mishina, M., Hayashi, T., 2018. Deficiency of AMPAR-Palmitoylation Aggravates Seizure Susceptibility. J. Neurosci. 38, 10220-10235.

Jiang, J., Suppiramaniam, V., Wooten, M.W., 2006. Posttranslational modifications and receptor-associated proteins in AMPA receptor trafficking and synaptic plasticity. Neurosignals 15, 266-282.

Jiao, X., Nawab, O., Patel, T., Kossenkov, A.V., Halama, N., Jaeger, D., Pestell, R.G., 2019. Recent Advances Targeting CCR5 for Cancer and Its Role in Immuno-Oncology. Cancer Res. 79, 4801-4807.

Kasama, T., Yajima, N., Matsukura, S., Adachi, M., 2006. Macrophage inflammatory protein 1 and CCR5 as attractive therapeutic targets 
for HIV infection. Recent Pat. Antiinfect. Drug Discov. 1, 275-280.

Kennedy, M.E., Limbird, L.E., 1993. Mutations of the alpha $2 \mathrm{~A}$-adrenergic receptor that eliminate detectable palmitoylation do not perturb receptor-G-protein coupling. J. Biol. Chem. 268, 8003-8011.

Kharbanda, A., Walter, D.M., Gudiel, A.A., Schek, N., Feldser, D.M., Witze, E.S., 2020. Blocking EGFR palmitoylation suppresses PI3K signaling and mutant KRAS lung tumorigenesis. Science Signaling 13. aax2364

Ko, P.-J., Dixon, S.J., 2018. Protein palmitoylation and cancer. EMBO Rep. 19. e46666

Kraft, K., Olbrich, H., Majoul, I., Mack, M., Proudfoot, A., Oppermann, M., 2001. Characterization of sequence determinants within the carboxyl-terminal domain of chemokine receptor CCR5 that regulate signaling and receptor internalization. J. Biol. Chem. 276, 34408-34418.

Lemonidis, K., Salaun, C., Kouskou, M., DiezArdanuy, C., Chamberlain, L.H., Greaves, J., 2017. Substrate selectivity in the zDHHC family of S-acyltransferases. Biochem. Soc. Trans. 45, 751-758.

Lin, D.-T., Makino, Y., Sharma, K., Hayashi, T., Neve, R., Takamiya, K., Huganir, R.L., 2009. Regulation of AMPA receptor extrasynaptic insertion by $4.1 \mathrm{~N}$, phosphorylation and palmitoylation. Nat. Neurosci. 12, 879-887.

Linder, M.E., Deschenes, R.J., 2007. Palmitoylation: policing protein stability and traffic. Nat. Rev. Mol. Cell Biol. 8, 74-84.

Liu, R., Wang, D., Shi, Q., Fu, Q., Hizon, S., Xiang, Y.K., 2012. Palmitoylation regulates intracellular trafficking of $\beta 2$ adrenergic receptor/arrestin/phosphodiesterase 4D complexes in cardiomyocytes. PloS One 7, e42658.

Lohse, M.J., Engelhardt, S., Eschenhagen, T., 2003. What Is the Role of $\beta$-Adrenergic Signaling in Heart Failure? Circulation Research 93, 896-906.

Loisel, T.P., Adam, L., Hebert, T.E., Bouvier, M., 1996. Agonist stimulation increases the turnover rate of beta 2AR-bound palmitate and promotes receptor depalmitoylation. Biochemistry 35, 15923-15932.
Marin, E.P., Jozsef, L., Di Lorenzo, A., Held, K.F., Luciano, A.K., Melendez, J., Milstone, L.M., Velazquez, H., Sessa, W.C., 2016. The Protein Acyl Transferase ZDHHC21 Modulates a1 Adrenergic Receptor Function and Regulates Hemodynamics.

Arteriosclerosis, Thrombosis, and Vascular Biology 36, 370-379.

Moffett, S., Rousseau, G., Lagacé, M., Bouvier, M., 2001. The palmitoylation state of the beta(2)-adrenergic receptor regulates the synergistic action of cyclic AMP-dependent protein kinase and beta-adrenergic receptor kinase involved in its phosphorylation and desensitization. J. Neurochem. 76, 269-279.

Mouillac, B., Caron, M., Bonin, H., Dennis, M., Bouvier, M., 1992. Agonist-modulated palmitoylation of beta 2-adrenergic receptor in Sf9 cells. J. Biol. Chem. 267, 2173321737.

Mueller, A., Kelly, E., Strange, P.G., 2002. Pathways for internalization and recycling of the chemokine receptor CCR5. Blood 99, 785-791.

Mumby, S.M., Kleuss, C., Gilman, A.G., 1994. Receptor regulation of G-protein palmitoylation. Proc. Natl. Acad. Sci. U.S.A. 91, 2800-2804.

Nguyen, D.H., Taub, D., 2002. Cholesterol is essential for macrophage inflammatory protein 1 beta binding and conformational integrity of CC chemokine receptor 5 . Blood 99, 4298-4306.

Nilsson, M.B., Le, X., Heymach, J.V., 2020. $\beta$ Adrenergic Signaling in Lung Cancer: A Potential Role for Beta-Blockers. J Neuroimmun. Pharmacol. 15, 27-36.

Ohno, Y., Kihara, A., Sano, T., Igarashi, Y., 2006. Intracellular localization and tissuespecific distribution of human and yeast $\mathrm{DHHC}$ cysteine-rich domain-containing proteins. Biochim Biophys Acta 1761, 47483.

Percherancier, Y., Planchenault, T., Valenzuela-Fernandez, A., Virelizier, J.L., Arenzana-Seisdedos, F., Bachelerie, F., 2001. Palmitoylation-dependent control of degradation, life span, and membrane expression of the CCR5 receptor. J. Biol. Chem. 276, 31936-31944.

Romano, R., Bucci, C., 2020. Role of EGFR in the Nervous System. Cells 9. e46666 
Runkle, K.B., Kharbanda, A., Stypulkowski, E., Cao, X.-J., Wang, W., Garcia, B.A., Witze, E.S., 2016. Inhibition of DHHC20-Mediated EGFR Palmitoylation Creates a Dependence on EGFR Signaling. Molecular Cell 62, 385396.

Salaun, C., Greaves, J., Chamberlain, L.H., 2010. The intracellular dynamic of protein palmitoylation. J. Cell Biol. 191, 1229-38.

Sanchooli, J., Sanadgol, N., Kazemi Arababadi, M., Kennedy, D., 2014. CCR5 plays important roles in hepatitis $B$ infection. Viral Immunol. 27, 2-6.

Savransky, V., Ionin, B., Reece, J., 2020. Current Status and Trends in Prophylaxis and Management of Anthrax Disease. Pathogens 9. 370.

Signoret, N., Pelchen-Matthews, A., Mack, M., Proudfoot, A.E.I., Marsh, M., 2000.

Endocytosis and Recycling of the HIV Coreceptor Ccr5. J. Cell Biol. 151, 12811294.

Sohn, H., Park, M., 2019. Palmitoylationmediated synaptic regulation of AMPA receptor trafficking and function. Arch. Pharm. Res. 42, 426-435.

Spinelli, M., Fusco, S., Mainardi, M., Scala, F., Natale, F., Lapenta, R., Mattera, A., Rinaudo, M., Li Puma, D.D., Ripoli, C., Grassi, A., D’Ascenzo, M., Grassi, C., 2017. Brain insulin resistance impairs hippocampal synaptic plasticity and memory by increasing GluA1 palmitoylation through FoxO3a. Nat. Commun. 8, 2009.

Sweeney, D.A., Hicks, C.W., Cui, X., Li, Y., Eichacker, P.Q., 2011. Anthrax Infection. Am. J. Respir. Crit. Care Med. 184, 13331341.

Termini, C.M., Gillette, J.M., 2017. Tetraspanins Function as Regulators of Cellular Signaling. Front. Cell Developmental Biol. 5, 34.

Tomas, A., Futter, C.E., Eden, E.R., 2014. EGF receptor trafficking: consequences for signaling and cancer. Trends Cell Biol. 24, 26-34.

Tonn Eisinger, K.R., Woolfrey, K.M., Swanson, S.P., Schnell, S.A., Meitzen, J., Dell'Acqua, M., Mermelstein, P.G., 2018. Palmitoylation of caveolin-1 is regulated by the same DHHC acyltransferases that modify steroid hormone receptors. J. Biol. Chem. 293, 15901-15911.
Tsutsumi, R., Fukata, Y., Noritake, J., Iwanaga, T., Perez, F., Fukata, M., 2009. Identification of $G$ protein alpha subunit-palmitoylating enzyme. Mol. Cell Biol. 29, 435-47.

Venkatesan, S., Rose, J.J., Lodge, R., Murphy, P.M., Foley, J.F., 2003. Distinct mechanisms of agonist-induced endocytosis for human chemokine receptors CCR5 and CXCR4. Mol. Biol. Cell 14, 3305-3324.

Vetrivel, K.S., Meckler, X., Chen, Y., Nguyen, P.D., Seidah, N.G., Vassar, R., Wong, P.C., Fukata, M., Kounnas, M.Z., Thinakaran, G., 2009. Alzheimer Disease $A \beta$ Production in the Absence of S-Palmitoylation-dependent Targeting of BACE1 to Lipid Rafts. J. Biol. Chem. 284, 3793-3803.

Wang, Y.-N., Hung, M.-C., 2012. Nuclear functions and subcellular trafficking mechanisms of the epidermal growth factor receptor family. Cell Biosci. 2, 13.

Warne, T., Serrano-Vega, M.J., Tate, C.G., Schertler, G.F.X., 2009. Development and crystallization of a minimal thermostabilised $\mathrm{G}$ protein-coupled receptor. Prot. Express. Purif. 65, 204-213.

Westermann, B., 2012. Bioenergetic role of mitochondrial fusion and fission. Biochimica et Biophysica Acta 1817, 1833-1838.

Won, S.J., Kit, M.C.S., Martin, B.R., 2018. Protein depalmitoylases. Critical Reviews in Biochemistry and Molecular Biology 53, 8398.

Wu, Y., Yoder, A., 2009. Chemokine Coreceptor Signaling in HIV-1 Infection and Pathogenesis. PLOS Pathogens 5, e1000520.

Xia, Z.-X., Shen, Z.-C., Zhang, S.-Q., Wang, J., Nie, T.-L., Deng, Q., Chen, J.-G., Wang, F., Wu, P.-F., 2019. De-palmitoylation by $\mathrm{N}$ (tert-Butyl) hydroxylamine inhibits AMPARmediated synaptic transmission via affecting receptor distribution in postsynaptic densities. C.N.S. Neurosci. Ther. 25, 187199.

Yang, G., Xiong, W., Kojic, L., Cynader, M.S., 2009. Subunit-selective palmitoylation regulates the intracellular trafficking of AMPA receptor. Eur. J. Neurosci. 30, 35-46.

Yang, X., Zheng, E., Ma, Y., Chatterjee, V., Villalba, N., Breslin, J.W., Liu, R., Wu, M.H., Yuan, S.Y., 2021. DHHC21 deficiency 
attenuates renal dysfunction during septic injury. Sci. Rep. 11, 11146.

Zmuda, F., Chamberlain, L.H., 2020.

Regulatory Effects of Post-Translational

Modifications on zDHHC S-acyltransferases.

J. Biol. Chem. 295,14640-14652.

Zuckerman, D.M., Hicks, S.W., Charron, G., Hang, H.C., Machamer, C.E., 2011.

Differential regulation of two palmitoylation sites in the cytoplasmic tail of the beta1adrenergic receptor. J. Bio.I Chem. 286, 19014-19023. 


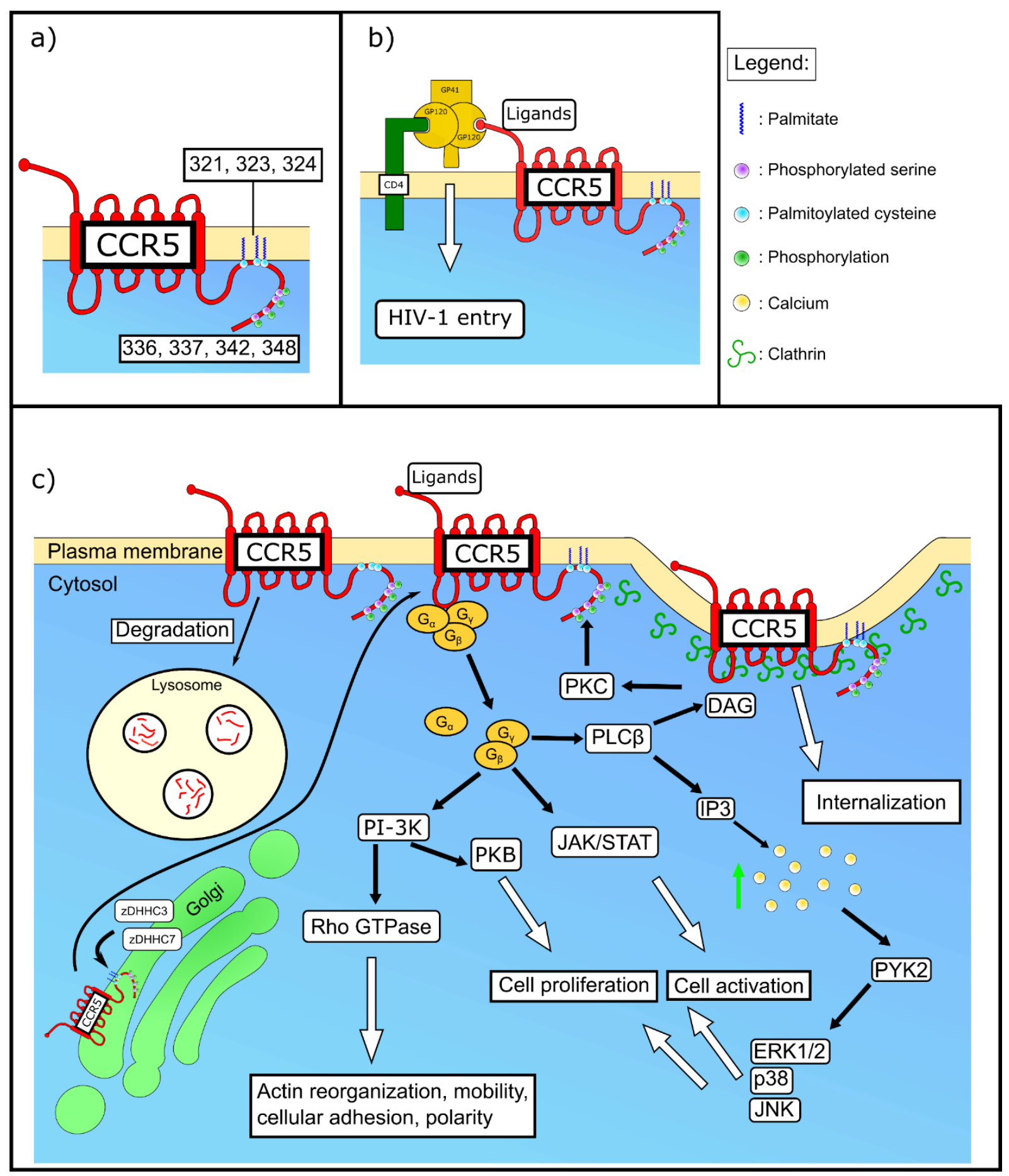

Figure 1. CCR5 palmitoylation, signalization and trafficking. (a) CCR5 topology, with its three palmitoylation and four phosphorylation sites. (b) CD4 and palmitoylated CCR5 interact with gp120 and gp41, the envelope proteins of HIV-1, enabling HIV-1 entry by fusion at the plasma membrane. (c) CCR5 is palmitoylated by DHHC3 and DHHC7 that localize to the Golgi apparatus. Unpalmitoylated CCR5 is retained in the Golgi or is quickly degraded in lysosomes following endocytosis. CCR5 interacts with $\mathrm{G}_{\alpha}, \mathrm{G}_{\beta}$ and $\mathrm{G}_{\gamma}$ at the plasma membrane. CCR5 ligand can be MIP-1a, CCL3L1, MIP-1b, RANTES, MCP-2, CCL11, MCP-4, or HCC-4. Upon ligand binding, $\mathrm{G}_{\alpha}$ dissociates from $G_{\beta \gamma}$, and 3 pathways are activated. The PI-3K pathway induces activation of Rho GTPases, causing actin reorganization, cellular adhesion, polarity and mobility, while PKB activation triggers cell proliferation. The JAK/STAT pathway induces cell activation. PLC $_{\beta}$ activation produces DAG, activating PKC, and inducing CCR5 phosphorylation. PLC $\beta$ also generates IP3, inducing intracellular release of calcium, PYK2, ERK1/2, p38, and JNK activation, that will induce cell proliferation and activation. Adapted from (Jiao et al., 2019) and (Sanchooli et al., 2014). 


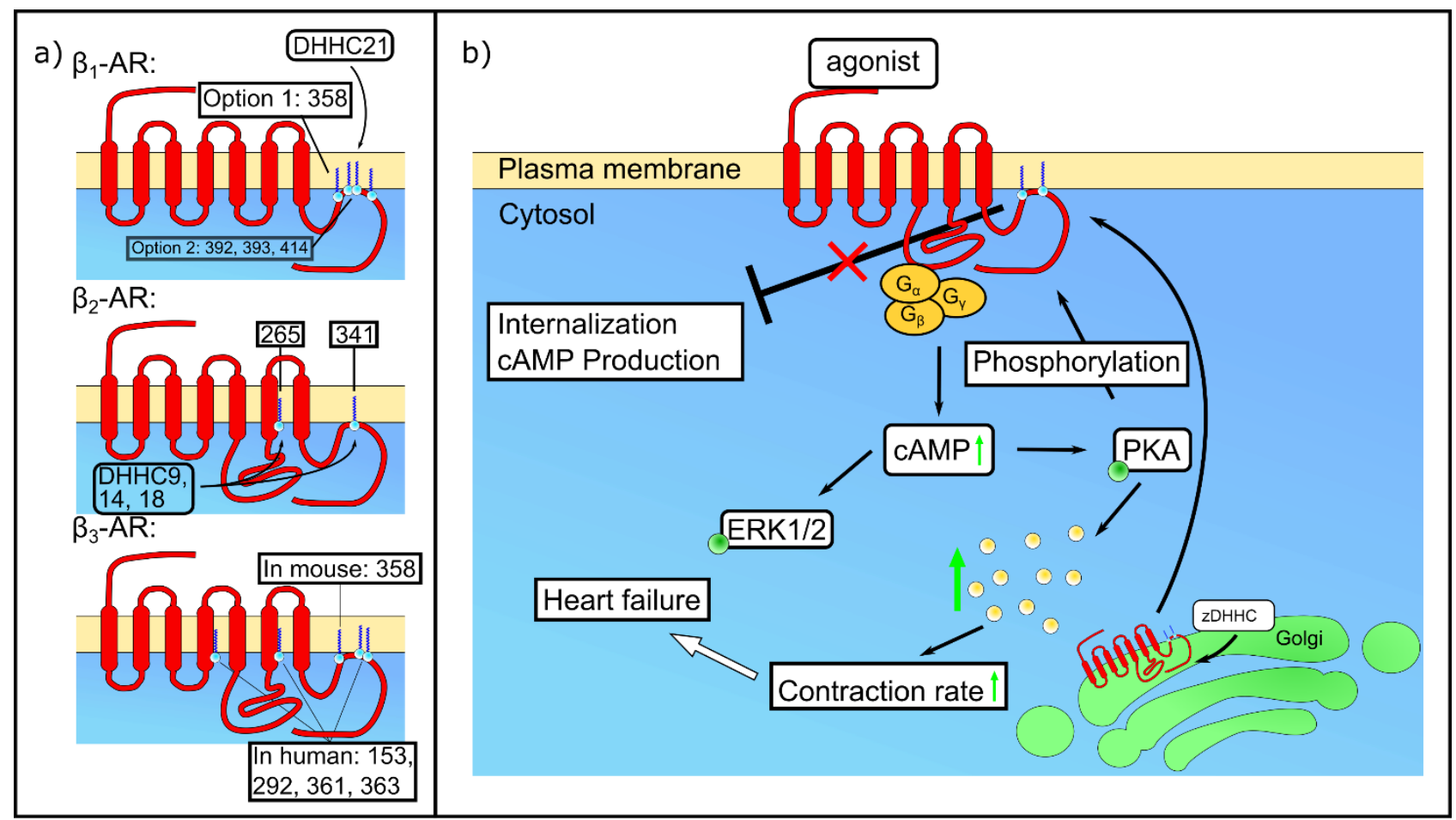

Legend:

: Palmitate $\quad$ :Phosphorylation $\quad \odot:$ Palmitoylated cysteine $\bigcirc:$ Calcium

Figure 2. Impact of $\beta$-AR palmitoylation on receptor signalization and internalization. (a)

Topology of $\beta_{1}-A R, \beta_{2}-A R$, and $\beta_{3}-A R$ with their palmitoylation sites and alternative positions. (b) In the Golgi, DHHC21 palmitoylates $\beta_{1}-\mathrm{AR}$, while DHHCs-9, -14 and-18 palmitoylate $\beta_{2}-\mathrm{AR}$. When the $\beta-A R$ is activated by an agonist such as isoproterenol, G-protein activation induces accumulation of cAMP, thereby triggering phosphorylation of ERK1/2 and PKA. Activated PKA induces phosphorylation of $\beta-A R$ and calcium release from intracellular stores, increasing heart contraction rate, potentially inducing heart failure. Depalmitoylation of $\beta$-AR inhibits its internalization and cAMP production. 


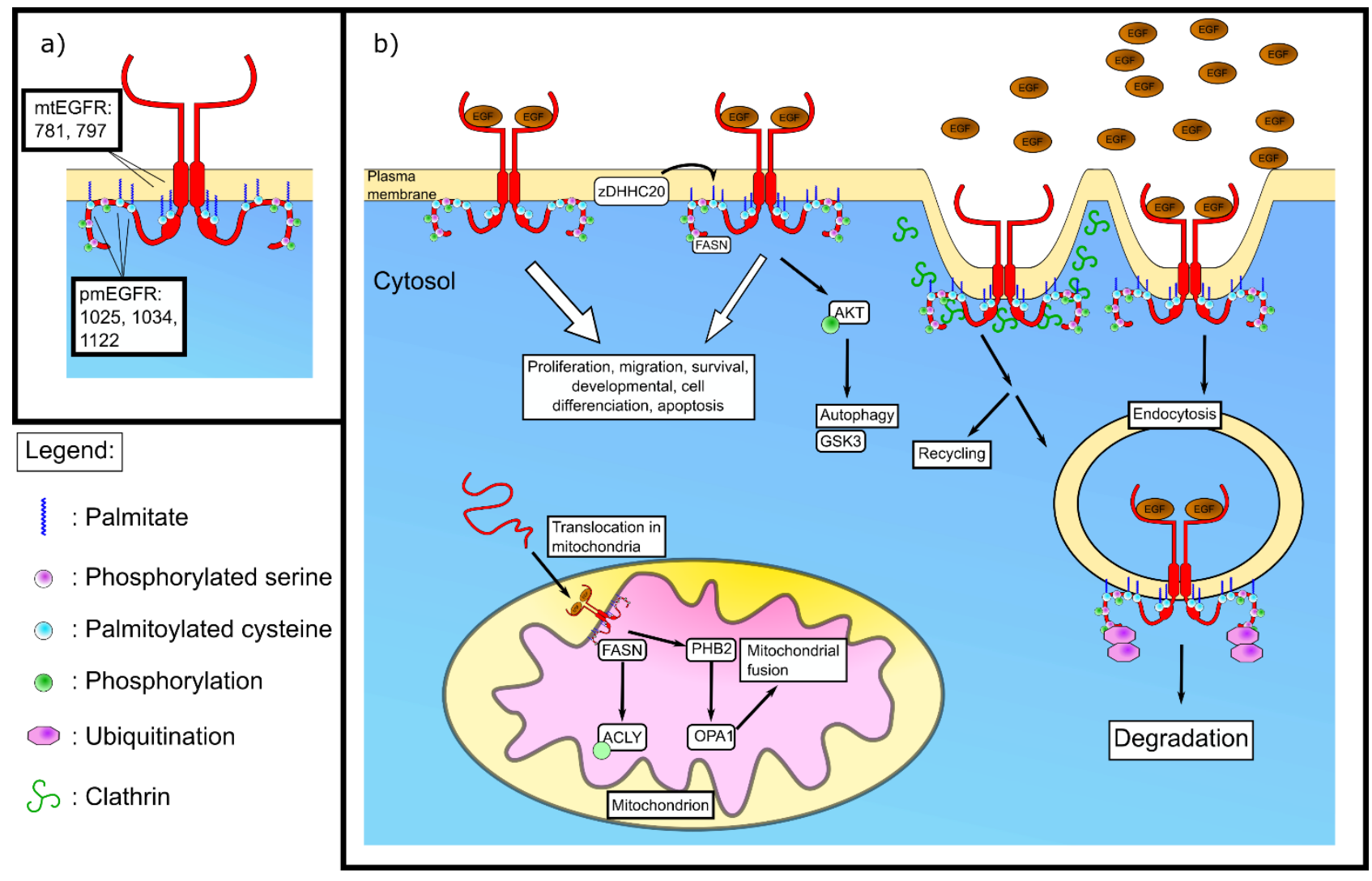

Figure 3. Effect of EGFR palmitoylation on receptor signalization and trafficking. (a) EGFR topology indicating the different palmitoylation sites for mtEGFR and pmEGFR. (b) pmEGFR is palmitoylated by DHHC20 at the plasma membrane. Palmitoylated EGFR interacts with FASN both at the plasma membrane and in mitochondria. In mitochondria, mtEGFR phosphorylates ATP citrate Lyase (ACLY) and induces PHB2 and OPA1, thereby promoting mitochondria fusion. At the plasma membrane, activation of pmEGFR by EGF promotes the phosphorylation of AKT that becomes activated, promoting autophagy and activation of GSK3. Unpalmitoylated EGFR triggers more efficient activation. EGFR is internalized via two pathways: clathrin-mediated endocytosis $(\mathrm{CME})$ in the presence of low concentrations of EGF and clathrin-independent endocytosis $(\mathrm{CIE})$ in the presence of high EGF concentrations. Following CME, EGFR can be recycled or ubiquitinated, inducing its degradation. Following CIE, most EGFR is directly ubiquitinated and degraded (Tomas et al., 2014). 


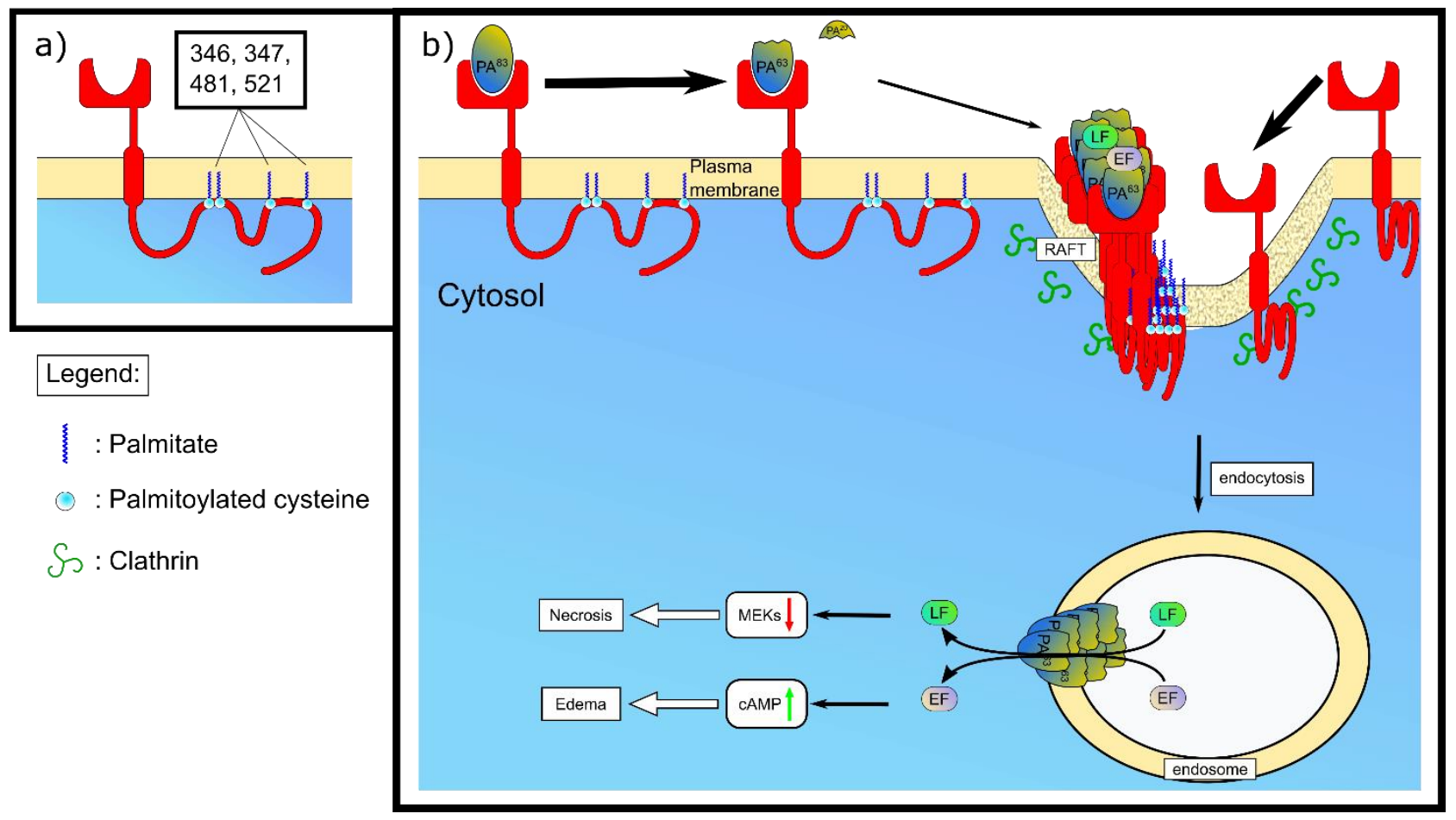

Figure 4. Palmitoylation of the anthrax receptors TEM8 and CMG2 affects their signalization and trafficking. (a) Topology of TEM8 and CMG2. The four palmitoylation sites of TEM8 are indicated. (b) Palmitoylated anthrax receptors bind to $\mathrm{PA}^{83}$, before cleavage of $\mathrm{PA}^{83}$ to produce $P^{63}$. PA ${ }^{63}$ binds to its receptor, to six other couples $P^{63}$-receptor, to LF and EF. This large complex is recruited by lipid rafts before endocytosis via CME. Within endosomes, the seven PA ${ }^{63}$ form a pore enabling delivery of LF and EF to the cytosol. EF produces CAMP, inducing edema, while LF cleaves MEKs inducing necrosis. Unpalmitoylated receptors more efficiently localize to lipid rafts, facilitating receptor endocytosis and degradation. 


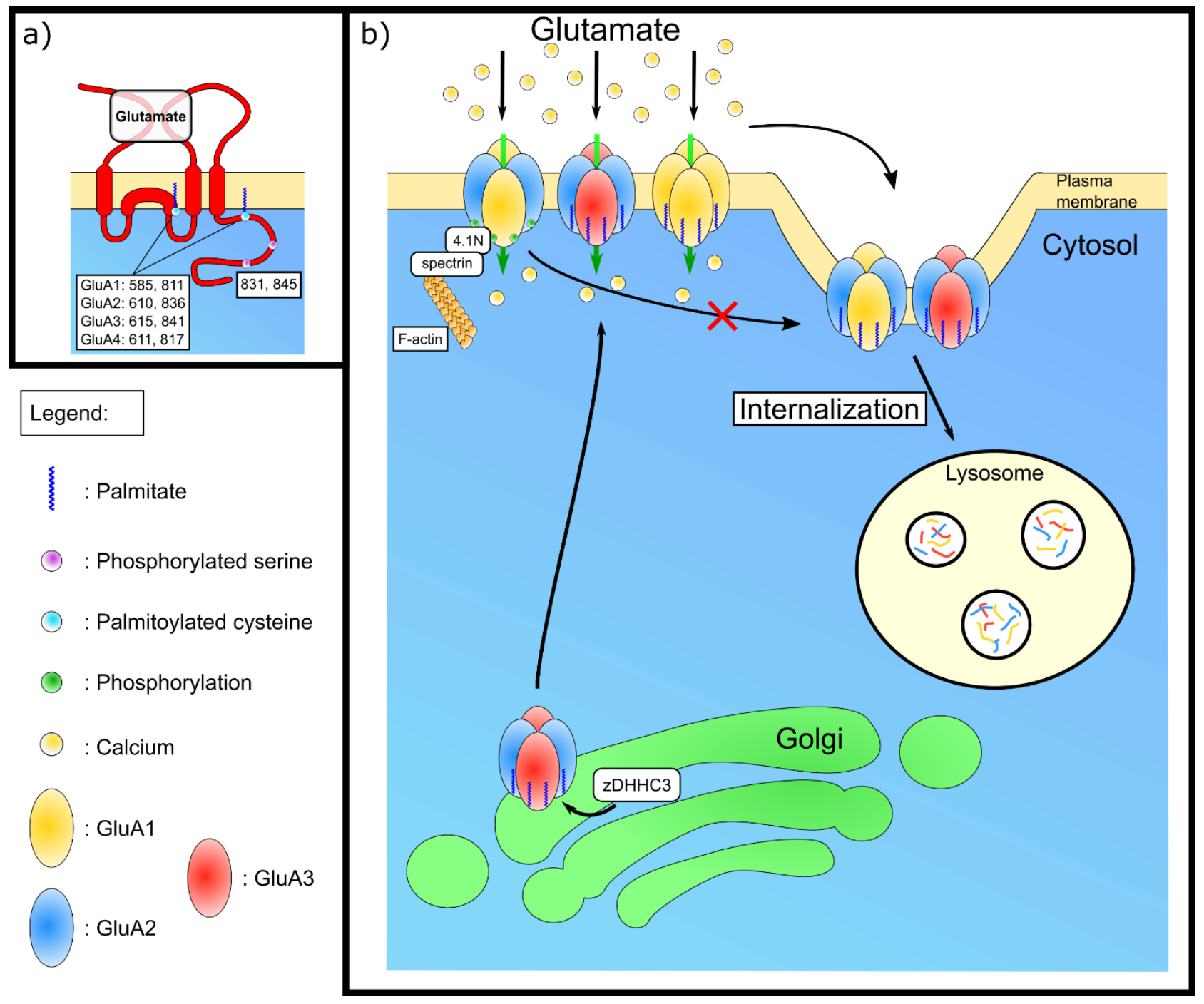

Figure 5. Impact of AMPAR palmitoylation on signalization and trafficking. (a) Topology and palmitoylation sites of each subunit of AMPAR: GluA1, GluA2, GluA3, and GluA4 (not depicted because expressed only during early development (Jiang et al., 2006)). (b) zDHHC3 (GODZ) induces palmitoylation of AMPAR and its accumulation in the Golgi, while depalmitoylation triggers transport to the plasma membrane. Surface palmitoylated AMPAR is susceptible to internalization and degradation within lysosomes, while unpalmitoylated AMPAR is resident at the synaptic membrane due to interaction with $4.1 \mathrm{~N}$, that itself interacts with spectrin and F-actin. 